\title{
Lépidic adenocarcinoma of the Lung Mimicking multiple nodules and pulmonary cysts
}

\author{
anass Khacha ${ }^{1}$, Mounia Serraj ${ }^{1}$, zineb yammouri ${ }^{1}$, nizar bouardi ${ }^{1}$, Meriem Haloua ${ }^{1}$, badre \\ eddine Alami ${ }^{1}$, moulay youssef alaoui lamrani ${ }^{1}$, Meryem Boubbou ${ }^{1}$, and mustapha \\ maaroufi $^{1}$ \\ ${ }^{1} \mathrm{CHU}$ Hassan II
}

November 20, 2021

\begin{abstract}
Lepidic growth adenocarcinoma is defined as tumor cells proliferating along the surface of intact alveolar walls without stromal or vascular invasion pathologically (1).

Lépidic adenocarcinoma of the Lung Mimicking multiple nodules and pulmonary cysts

A. Khacha ${ }^{1}$.M.Serraj ${ }^{2}$. Z. Yammouri ${ }^{1}$. N. Bouardi ${ }^{1}$.M.Haloua ${ }^{1}$.Y. Alaoui Lamrani ${ }^{1}{ }^{\text {M. Boubbou }}{ }^{1}$. F. Belahsen. M. Maaroufi ${ }^{1}$ B. Alami ${ }^{1}$

1 Radiology department of CHU HASSAN II de Fez, Morocco, Faculty of medecine Fez, University Sidi Mohamed Ben Abdellah, Fez, Morocco

2 Pneumology department of CHU HASSAN II de Fez, Morocco, Faculty of medecine Fez, University Sidi Mohamed Ben Abdellah, Fez, Morocco
\end{abstract}

No conflict of intrest

Abstract

Lepidic growth adenocarcinoma is defined as tumor cells proliferating along the surface of intact alveolar walls without stromal or vascular invasion pathologically (1). Travis et al . (2) proposed a classification of lung adenocarcinoma based on the International Association for the Study of Lung Cancer (IASLC), the American Thoracic Society (ATS), and the European Respiratory Society (ERS) recommendations. They did not use the terms bronchioloalveolar carcinoma or mixed subtype, and they classified adenocarcinoma in situ (AIS) as pure lepidic growth, and minimally invasive adenocarcinoma (MIA) as predominant lepidic growth containing invasion foci of less than $5 \mathrm{~mm}$ for small (less than $3 \mathrm{~cm}$ ) solitary adenocarcinomas.In addition, invasive lepidic predominant adenocarcinoma (LPA) was defined as a lepidic growth containing invasion foci of more than $5 \mathrm{~mm}$. They described that solitary adenocarcinomas with pure lepidic growth, termed "AIS".

We report the case of a young patient who had no medical history and presented to the ER with acute dyspnea and cough.

\section{Introduction:}

Lepidic-predominant adenocarcinoma (LPA) of the lung, formerly known as non-mucinous bronchoalveolar carcinoma, is a subtype of invasive adenocarcinoma of the lung identified histologically when the lepidic component comprises the majority of the lesion. 


\section{Case report}

A 20 -year-old nonsmoker young man with no significant medical or surgical history. There was also no history of high-risk sexual behavior and no history of drug addiction. He presented to the emergency room with a grade III dyspnea and non productive cough starting in the last two months with aggravation in the last 2 days becoming grade IV dyspnea associated to loss of weight ( $5 \mathrm{Kg}$ during the last week) and fever. Physical examination showed tachycardia $120 \mathrm{~b} / \mathrm{min}$, and temperature of $38,5^{\circ}$. Respiratory frequency was as 22 cycles/min and saturation measured at $81 \%$.

The patient was fully conscious with normal neurological examination.

Blood examination showed polyglobulia Hematocrite level at $51 \%$ and hemoglobine level at $18 \mathrm{~g} / \mathrm{dl}$.

BK simples were negative.

Broncho-alveolar lavage was positive for pneumocytosis.

HIV blood test was negative.

The patient received oxygenotherapy (10L) and was initially placed on airborne precautions.

A chest plan radiographe showed multiples nodules(Figure1) that were confirmed on a chest CT afterwords with diffuse nodules some of theme were excavated nodules associated to some cystic lesions(Figure 2).

Through these results we concluded to a pulmonary pneumocytosis, and the patient was released and had a controle after 2 weeks.

The patient was re-adimitted to the emergency room five days later with aggravated respiratory symptoms a new chest CT was performed showing consolidation in lung bases(Figure 4), we decides to perform a CT guided percutaneous lung biopsy(Figure 5) that was in favour of an invasive lung adenocarcinoma with lepidic growth pattern(Figure 6 and 7). The patient died on the $6^{\text {th }}$ day of his second hospitalisation from respiratory distress.

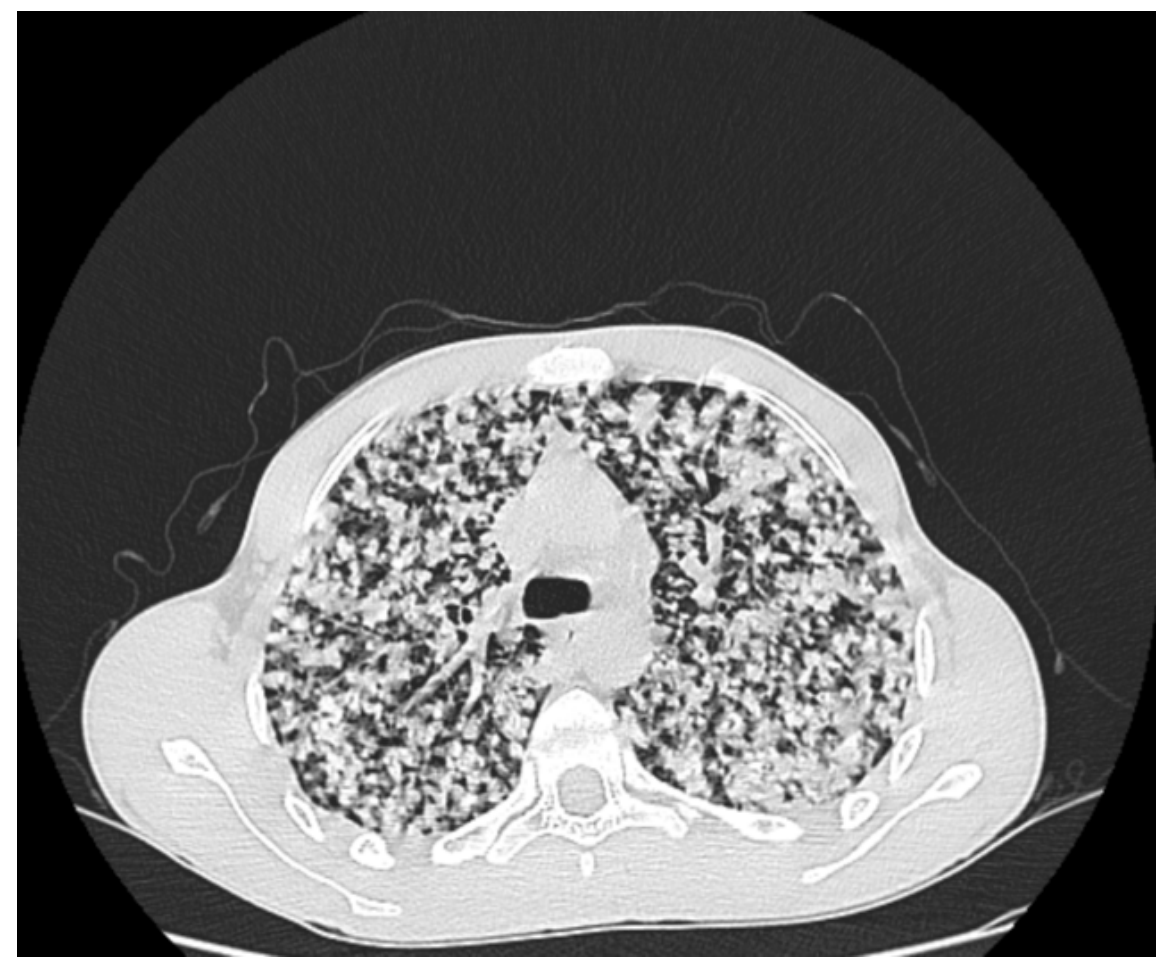


Figure 2 axial chest CT showing diffuse nodular and micnrodules with no predominance pattern

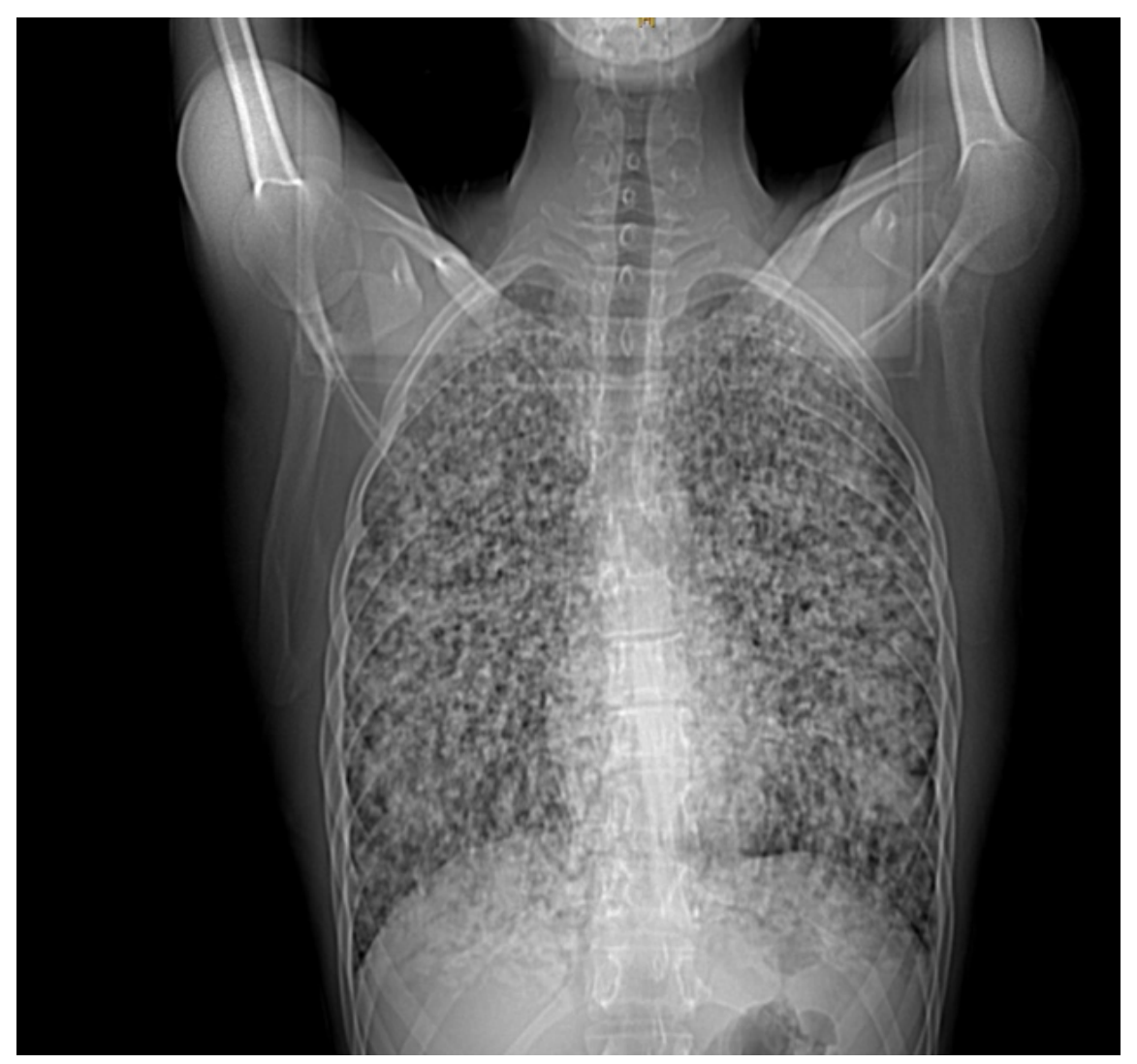

Figure 1 : plan chest radiographe showing diffuse reticular and nodulare predominant pattern. 


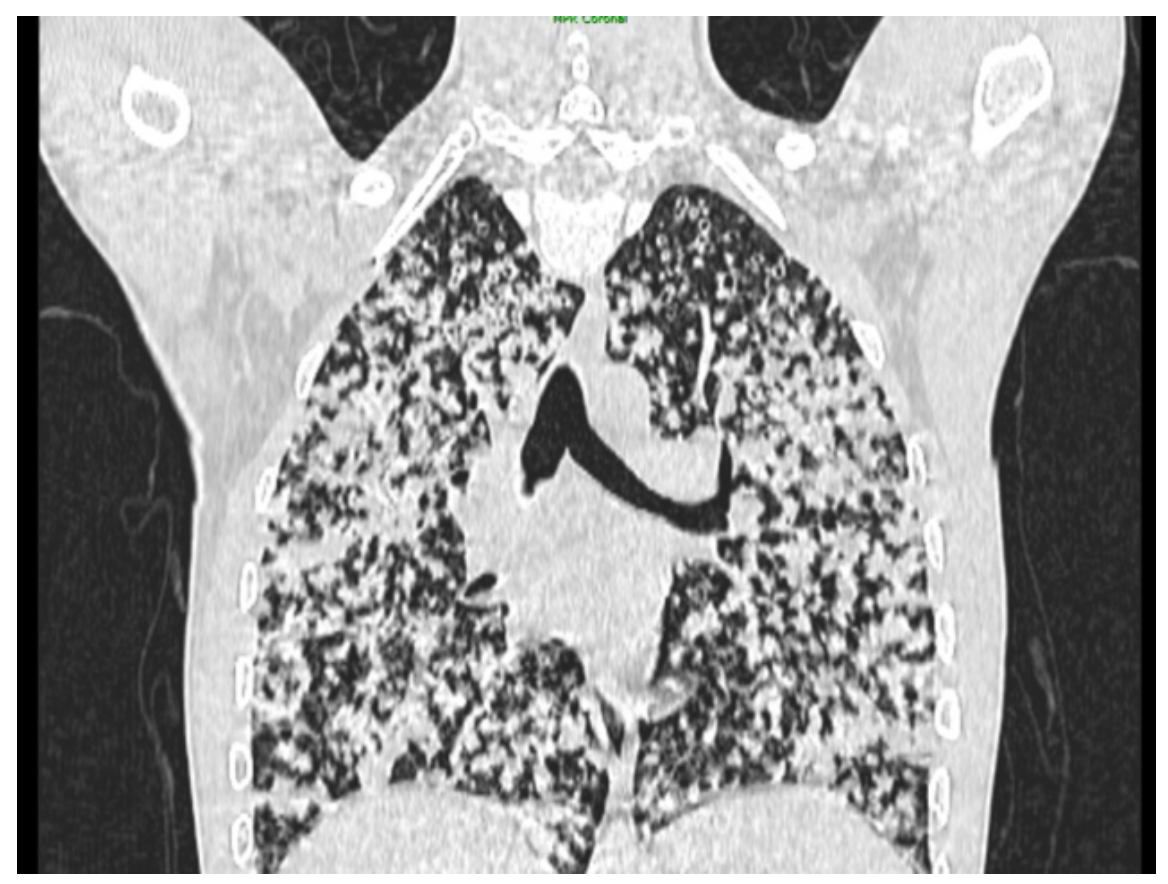

Figure 3 : coronal chest CT showing diffuse nodular and micnrodules with no predominance pattern

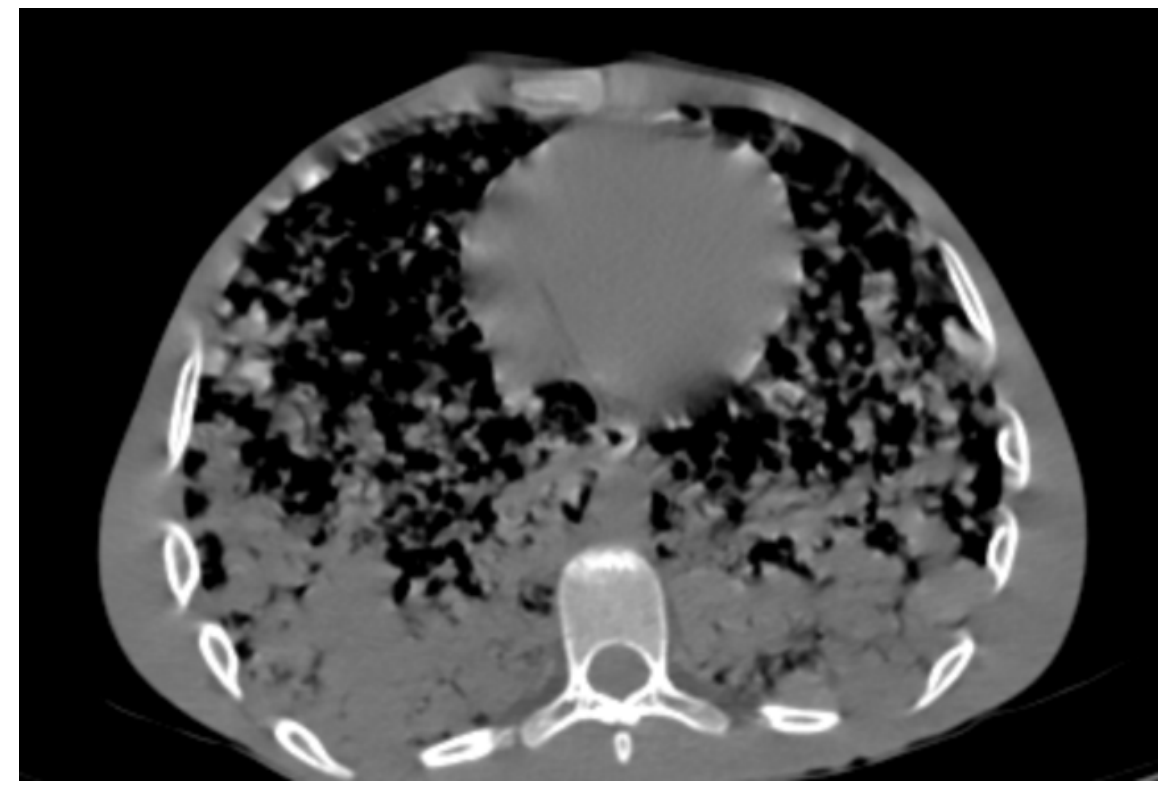

Figure 4 axial ches CT showing nodules and basal bilateral consolidation 


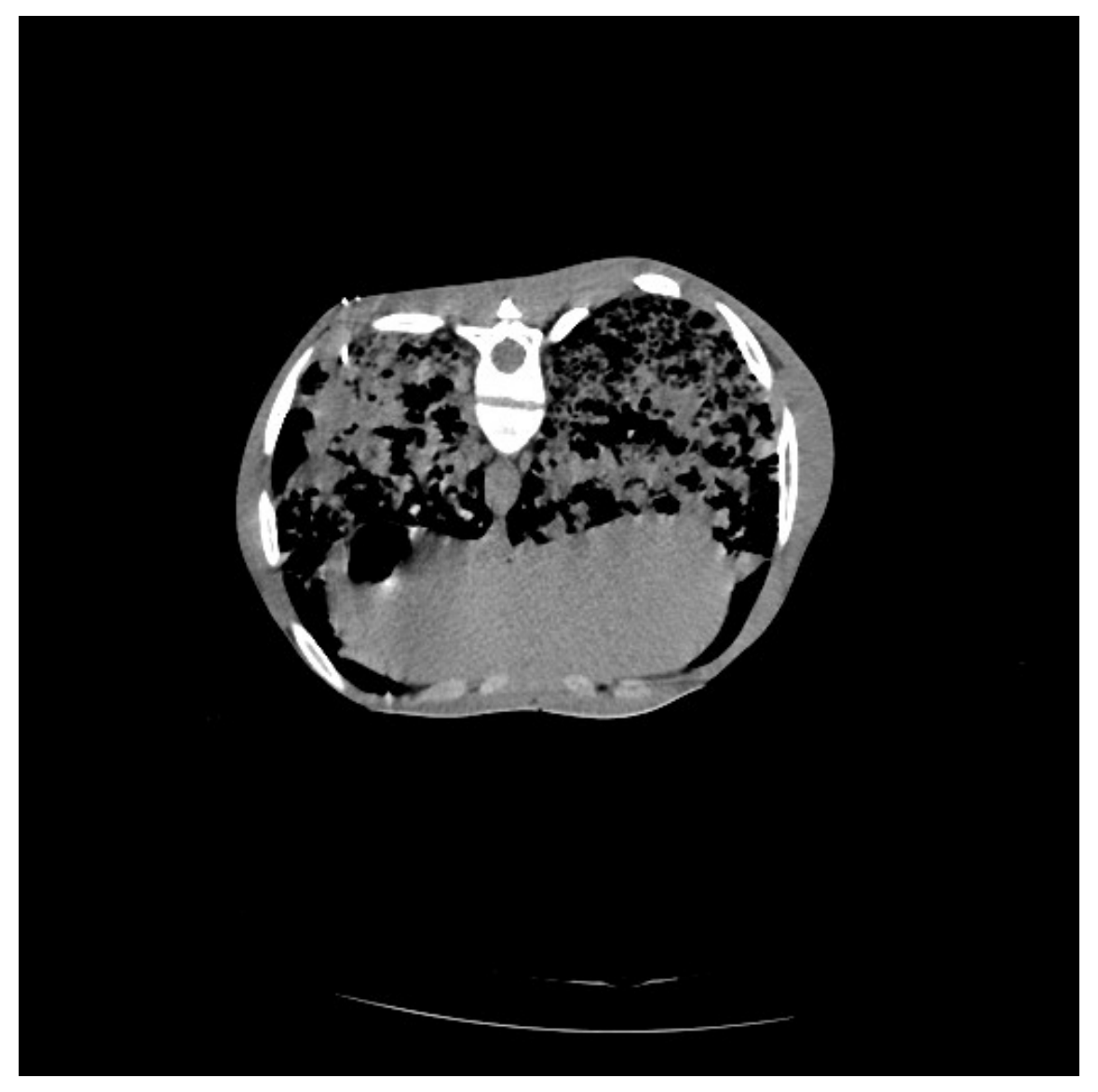

Figure 5 scan guided biopsy of right basal consolidation 


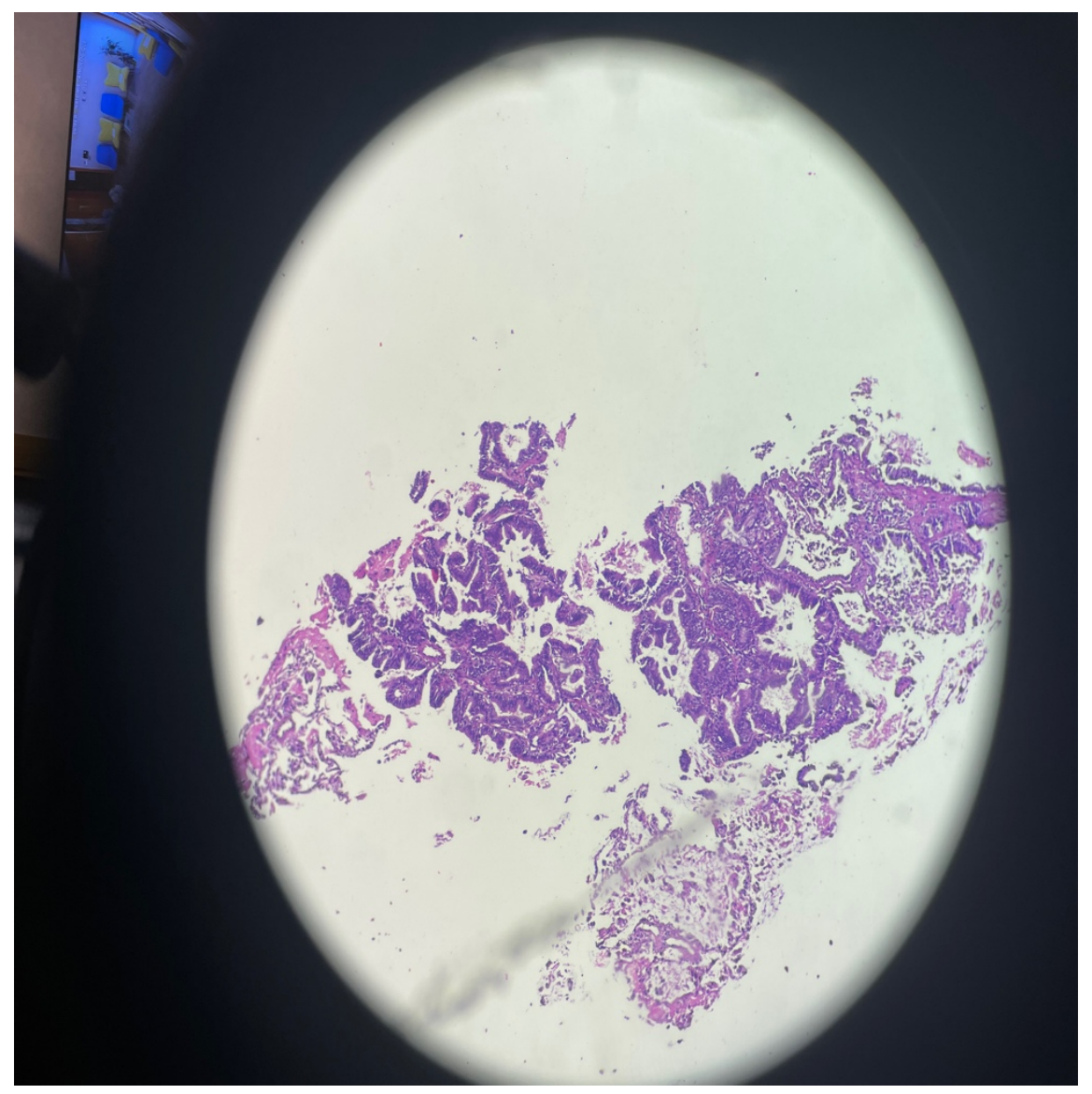

Figure 6 Adénocarcinome with lepidic arcitercture:

Tumor proliferation with atypical cells in the alveolar strcutures in cohesive pattern (x 10). 


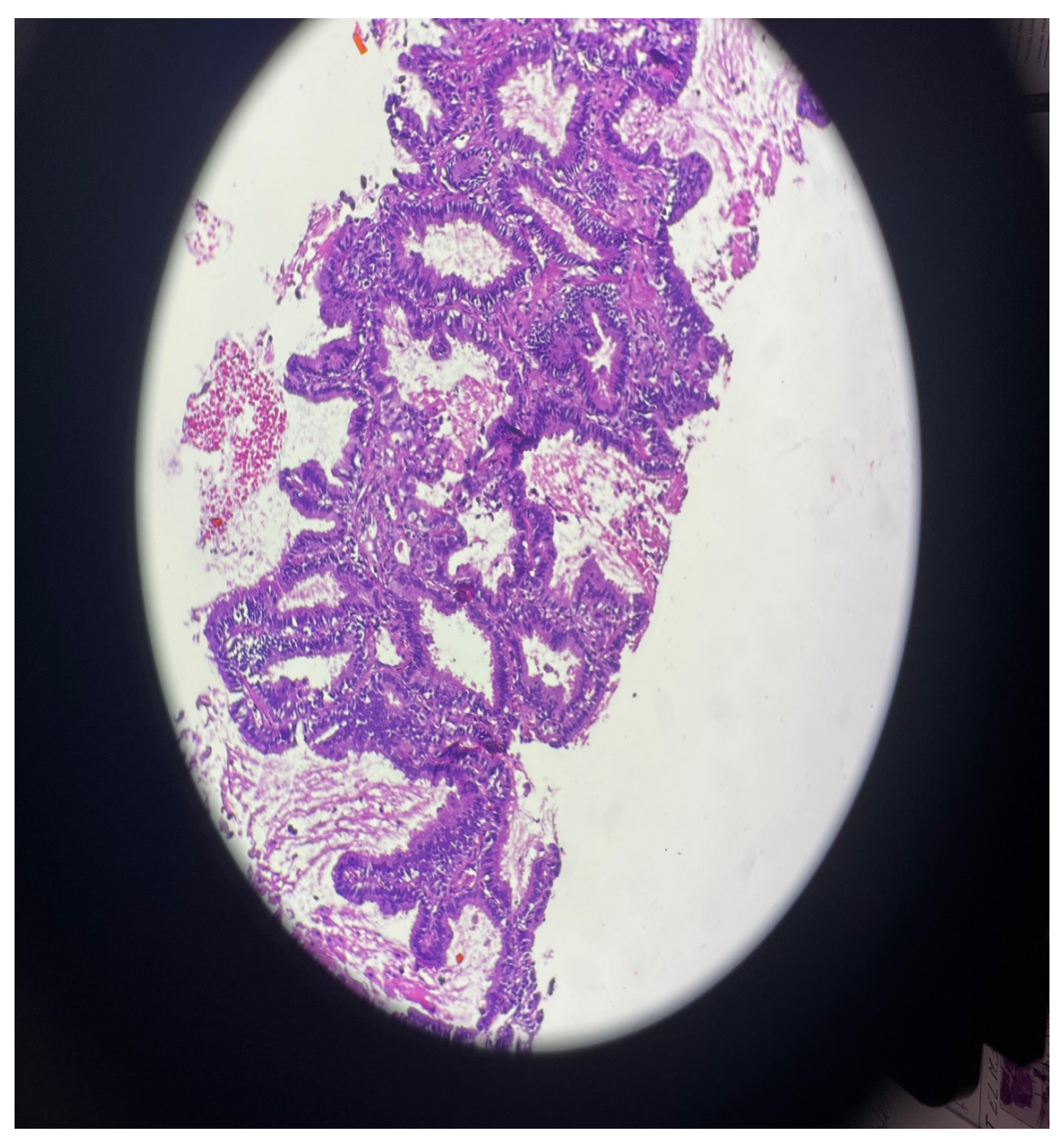

Figure 7 Adénocarcinome with lepidic arcitercture:

Tumor proliferation with atypical cells in the alveolar strcutures in cohesive pattern (x 20).

\section{Discussion}

Lung cancer is among the most frequently diagnosed cancers and the world's leading cause of cancer-related death [3]. The peak-incidence age for lung cancer is between 55 and 65 years, and smoking is a well-established risk factor: active and passive smokers have a 13-fold and 1.5-fold higher risk than nonsmokers, respectively [4]. Lung cancers are classified into Small Cell Lung Cancer (SCLC) and Non Small Cell Lung Cancer (NSCLC). NSCLCs are further divided into three major subtypes: squamous cell carcinoma, adenocarcinoma, and large cell carcinoma [3-5]. Squamous cell carcinoma and SCLC quite often present as a central mass [6].

Lung adenocarcinomas typically arise from the bronchial epithelium or bronchial glandular epithelium, and they are the most common type among nonsmokers and women $[3,5]$. Pathologically, adenocarcinoma may display an acinar, papillary, micropapillary, lepidic, or solid growth pattern, with either mucin or pneumocyte marker expression, and it is usually located peripherally with pleural involvement [6]. The most frequent radiological patterns are ground-glass, part-solid, and solid nodules on chest CT scans [7]. Primary lung cancer rarely presents as multiple nodules [8-9].

Multiple nodules in the lung are seen in a wide variety of conditions. The nodular pattern and excavated nodules could be associated to vasculitis such as Wegener and shurg strauss it could also be miliary TB, histoplasmosis, sarcoidosis, pneumoconiosis, bronchoalveolar carcinoma, or pulmonary siderosis, secondary 
metastasis to the lungs from primary cancers of the thyroid, kidney, and trophoblasts as well as some sarcomas $[2,3]$ High-resolution CT can help to narrow down the differential diagnoses by distributing the micronodules into centrilobular, perilymphatic, and random patterns. Infectious bronchiolitis commonly presents a centrilobular pattern and sarcoidosis a perilymphatic pattern, whereas a random pattern is suggestive of hematogenous metastases. [10]. A thorough review of the literature revealed a few similar cases reported as multiple nodules primary lung carcinoma [2]. The largest case series is reported from Japan, in which 5 such patients were included [3]. That report concluded that the prevalence of the multiple nodules phenotype is $<1 \%$ among japanese patients and had poor prognosis.

Khan et al.(11)also showed a 35 year old man with same clincal presentation and mutiple nodules diasgnosed as lepidic adenocarcinoma after lung biopsy.

Wigger et al reported the case of a 66 year-old female patient actively smoking (50 pack-year) with a history of respiratory bronchiolitis-ILD chest CT findings included bilateral, apical ground glass opacities ,numerous enlarging sub-centimeter upper lobe nodules and cysts adenocarcinoma was found in the lung biopsy in the follow up of the patient.(15)

On the other hand Farooqi et al (12) identified 26 (3.7\%) lesions that were abutting lung cysts and were later on identified as lepidic adenocarcinomas .

In a more recent retrospective study, Fintelmann et al (13) identified and assessed 30 lung cancers from a total of 2954 primary lung cancers diagnosed at their institution. In these 30 cases, cysts were in or adjacent to lung adenocarcinomas at some point leading up to the histologic diagnosis.

In many screening studies, the findings in cohorts of patients with missed cancers associated with cystic airspaces are highlighted. However, there is a lack of data regarding the percentages of these lesions that are ultimately diagnosed as malignant, and this paucity should prompt pro- spective research trials. Guo et al (14) provided minimal insight, with $4.7 \%$ of resected cystic lesions reflecting cancer in their study. However, this percentage cannot be extrapolated to lesions identified at imaging. In addition, these data are limited to patients who were physiologically capable of undergoing surgical resection and reflect an unusually large rate of benign cyst resections.

\section{Conclusion :}

Multiple nodules and air space cyst on chest imaging could be a presentation of many deseases such as histiocytosis, TB, fungal infections, sarcoidosis, and occupational lung disease. It can also be a manifestation of hematogenous metastasis to the lungs from thyroid and renal cancers, sarcomas. Adenocarcinoma of the lung, although rarely presenting as multiple nodules or cysts can lead to a delay in diagnosis. We conclude that adenocarcinoma of the lung may be considered a rare but sinister differential diagnosis of multiple nodules and air space cysts on chest imaging. It has a favorable response to tyrosine kinase inhibitorbased therapy even with brain metastasis.

\section{Consent statement:}

I confirm that all authors have confirmed to the submission.

Patient has passed away.

References

1. 1. Jones KD. Whence lepidic?: the history of a Canadian neologism. Arch Pathol Lab Med 2013;137:1822-.

2 Noguchi M, Morikawa A, Kawasaki M, et al. Small adenocarcinoma of the lung. Histologic characteristics and prognosis. Cancer 1995;75:2844-52. 10.1002/1097-0142(19950615)75:12<2844::AIDCNCR2820751209>3.0.CO;2-\# 
3. Torre LA, Bray F, Siegel RL, Ferlay J, Lortet-Tieulent J, Jemal A. Global cancer statistics, 2012. CA Cancer J Clin. 2015;65(2):87-108.

41 Jayaram Subhashchandra B, Ismailkhan M, Chikkaveeraiah Shashidhar K, Gopalakrishna Narahari M. A rare case of non-small cell carcinoma of lung presenting as miliary mottling. Iran J Med Sci. 2013;38(1):65-8.

5. Couraud S, Zalcman G, Milleron B, Morin F, Souquet PJ. Lung cancer in never smokers - a review. Eur J Cancer. 2012;48(9):1299-311.

6. Pillai S, Khan A, Khan S. Adenocarcinoma of the lung presenting with intrapulmonary miliary metastasis. Cureus. 2019;11(8):e5430.

7. Diederich S, Wormanns D, Semik M, Thomas M, Lenzen H, Roos N, et al. Screening for early lung cancer with low-dose spiral CT: prevalence in 817 asymptomatic smokers. Radiology. 2002;222(3):773-81.

8. Henschke CI, McCauley DI, Yankelevitz DF, Naidich DP, McGuinness G, Miettinen OS, et al. Early Lung Cancer Action Project: overall design and findings from baseline screening. Lancet. 1999;354(9173):99-105

9. Fachinger P, Tini GM, Grobholz R, Gambazzi F, Fankhauser H, Irani S. Pulmonary tularaemia: all that looks like cancer is not necessarily cancer - case report of four consecutive cases. BMC Pulm Med. 2015;15:27.

10. Kimmig L, Bueno J. Miliary nodules: not always tuberculosis. Ann Am Thorac Soc. 2017;14(12):1858-60.

11. Khan et al.: Adenocarcinoma of the Lung and Miliary Shadows.

12.Farooqi AO, Cham M, Zhang L, et al. Lung cancer associated with cystic airspaces. AJR Am J Roentgenol 2012;199(4):781-786.

13. Fintelmann FJ, Brinkmann JK, Jeck WR, et al. Lung cancers associated with cystic airspaces: natural history, pathologic correlation, and mutational analysis. J Thorac Imaging 2017;32(3):176-188.

14. Guo J, Liang C, Sun Y, Zhou N, Liu Y, Chu X. Lung cancer presenting as thin-walled cysts: an analysis of 15 cases and review of literature. Asia Pac J Clin Oncol 2016;12(1):e105-e112.

15. gregory Wigger,Eric Flenaugh,Gabriela oprean-llies,JordanKempker,Lucian Marts Fellow case reporters $10.1016 /$ j.chest.2020.08.1252 